\title{
DETERMINAÇÃO DE RISCO NUTRICIONAL E DESNUTRIÇÃ̃O POR ANTROPOMETRIA EM CRIANÇAS E ADOLESCENTES COM CIRROSE
}

\author{
Ana Cláudia Reis SCHNEIDER, Raquel Borges PINTO e Themis Reverbel da SILVEIRA
}

\begin{abstract}
RESUMO - Racional - A desnutrição é achado freqüente em adultos com cirrose, mas a prevalência de risco nutricional e de desnutrição é pouco conhecida em pacientes pediátricos. Objetivo - Avaliar a ocorrência de risco nutricional e desnutrição em pacientes pediátricos com cirrose atendidos regularmente no Setor de Gastroenterologia Pediátrica do Hospital de Clínicas de Porto Alegre, RS. Métodos - Estudo transversal com 42 crianças e adolescentes cirróticos com idades entre 3 meses e 18 anos. O estado nutricional foi determinado por escores $\mathrm{Z}$ de peso para idade, estatura para idade, índice de massa corporal e percentis para a prega cutânea tricipital e circunferência muscular do braço. Consideraram-se pacientes em risco nutricional aqueles com escore $\leq-1,28 \mathrm{Z}$, correspondente ao percentil $\leq 10$, e desnutridos aqueles com escore $\leq-2,0 \mathrm{Z}$, correspondente ao percentil $\leq 3$. Segundo o critério de Child-Pugh, 22 pacientes foram classificados como A (leve intensidade), 15 como B (moderada) e 5 como C (grave). Resultados - As médias e desvios-padrão dos escores $Z$ de peso para idade, estatura para idade e índice de massa corporal foram respectivamente - $0,38 \pm 1,4$ DP, - $0,83 \pm 1,16$ DP e $0,17 \pm 1,3$ DP. A prega cutânea tricipital e a circunferência muscular do braço apresentaram medianas no percentil 25. Encontraram-se em risco nutricional 3/42 pacientes (peso para a idade), 8/42 (estatura para idade), 12/37 (prega cutânea tricipital), 9/37 (circunferência muscular do baço) e 2/38 (índice de massa corporal); desnutridos $6 / 42$ (peso para a idade), 7/42 (estatura para idade), 4/37 (prega cutânea tricipital) e 4/37(circunferência muscular do braço) e 3/38 (índice de massa corporal). Conclusão - Os índices antropométricos mais comprometidos foram a relação estatura para idade e a prega cutânea tricipital. Ocorreu uma prevalência de 32,4\% de risco nutricional determinado pela prega cutânea tricipital e 16,7\% de desnutrição pela relação estatura para idade. A prega cutânea tricipital foi o indicador que melhor refletiu a presença de risco nutricional e o índice estatura para idade a desnutrição.
\end{abstract}

DESCRITORES - Transtornos da nutrição infantil. Estado nutricional. Antropometria. Cirrose hepática. Adolescente. Criança.

\section{INTRODUÇÃO}

O fígado é um órgão importante no metabolismo, armazenamento e distribuição de nutrientes, portanto a ocorrência de alterações nutricionais na doença hepática crônica é bastante comum. Os problemas nutricionais dos cirróticos são multifatoriais e além da ingestão inadequada de nutrientes, ocorrem ainda problemas de hipermetabolismo ${ }^{(4)}$, digestão e absorção ${ }^{(20,29)}$. Recentemente, CARVALHO e PARISE ${ }^{(8)}$ avaliaram o estado nutricional de 300 pacientes cirróticos adultos e encontraram sinais de desnutrição protéico-calórica em 75,3\% dos pacientes avaliados. Outros estudos realizados em nosso país demonstraram que a ingestão calórico-protéica, assim como a proporção de nutrientes, costuma ser inadequada tanto em hepatopatas adultos ${ }^{(15)}$ como em crianças $^{(2)}$ e estes fatores predispõem à desnutrição associados à disfunção hepática. Estudos que avaliam o estado nutricional de pacientes cirróticos são mais freqüentes em adultos, alcoólicos ou não ${ }^{(1,5,7,8,10,19,24,28)}$, porém há poucos dados de estudos com crianças e adolescentes com hepatopatias crônicas e estes são limitados, devido principalmente ao número pouco expressivo de pacientes ${ }^{(6,9,14,21,31)}$. A observação das alterações nutricionais são especialmente importantes quando ocorrem em crianças e adolescentes, que estão em fase de crescimento e desenvolvimento intensos, portanto pacientes pediátricos com doenças hepáticas crônicas devem ter seu crescimento constantemente monitorados e o risco nutricional prontamente identificado ${ }^{(29)}$. A deteç̧ão de sinais precoces de comprometimento nutricional pode evitar o aumento de sua gravidade e as seqüelas da desnutrição na vida adulta.

Dentre os métodos utilizados para avaliação nutricional temos: a avaliação subjetiva global e a dinamometria do aperto de mão ${ }^{(1)}$, que não foram validados para crianças; a análise por bioimpedância, que no caso da doença hepática é afetada pela flutuação na hidratação do paciente; a absormetria por emissão de duplo raio-X (DEXA), que é considerado o padrão-ouro para avaliação da composição corporal, contudo tem o seu uso limitado pelo alto custo e está disponível apenas em centros especializados.

Setor de Gastroenterologia Pediátrica - Departamento de Pediatria do Hospital de Clínicas de Porto Alegre, RS.

Financiamento: FIPE e CAPES

Correspondência: Dra. Themis Reverbel da Silveira - Rua 24 de Outubro, 1181 - Auxiliadora - 90510-003 - Porto Alegre, RS. E-mail: tsilveira@hcpa.ufrgs.br 
A antropometria, pela facilidade de execução, inocuidade e baixo custo, além da obtenção rápida dos resultados, tem se revelado como o método isolado mais utilizado para o diagnóstico nutricional, sobretudo na infância e adolescência ${ }^{(30)}$.

Este estudo teve como objetivo avaliar o estado nutricional de pacientes pediátricos com cirrose, com uso de antropometria, para determinar a prevalência de risco nutricional e desnutrição.

\section{MÉTODOS}

Foi realizado um estudo transversal, que avaliou, no período de agosto de 2003 a julho de 2005, 42 pacientes com cirrose atendidos regularmente no Ambulatório de Gastroenterologia Pediátrica do Hospital de Clínicas de Porto Alegre (HCPA), RS. Dos participantes do estudo, 37 eram de ambulatório e 5 estavam hospitalizados para realização de exames. Todos os pacientes eram compensados, lúcidos, sem edema e/ou ascite perceptível ao exame físico, no entanto, em oito a ascite de pequena intensidade foi comprovada por ecografia abdominal, realizada a não mais de 30 dias da data da coleta de dados.

Foram averiguados os fatores causa da cirrose, a presença de colestase e a classificação de gravidade da doença, determinada pelo escore de Child-Pugh ${ }^{(25)}$. A doença de leve intensidade é classificada como A, moderada B e severa C. Os exames para investigar a presença de colestase e determinar a gravidade da cirrose foram solicitados no mesmo período da avaliação antropométrica. Para determinação da causa da hepatopatia crônica, foram realizados exames para investigação de hepatites virais (HbsAg, IgM anti$\mathrm{HBc}$, anti-Hbc total, anti-HCV), anticorpos anti-músculo liso, anti-LKM, fator anti-nuclear, ceruloplasmina, cobre urinário, eletrólitos no suor, dosagem sérica de $\alpha 1$-antitripsina, pesquisa de alelos PiZZ e triagem para erros inatos do metabolismo. Os pacientes cuja causa da cirrose não pôde ser definida foram considerados como criptogênicos.

O diagnóstico de cirrose foi obtido por confirmação histopatológica (alteração da arquitetura do parênquima hepático com presença de formação nodular e fibrose na biopsia hepática) e na ausência desta, por exames laboratoriais (alterações de coagulação, hipoalbuminemia), ultra-sonográficos (fígado com ecogenicidade heterogênea, nódulos regenerativos e sinais de hipertensão portal) e/ou exames endoscópicos (presença de varizes gastroesofágicas e/ou gastropatia hipertensiva porta).

A presença de colestase foi definida pelo seguinte critério da Sociedade Norte Americana de Gastroenterologia, Hepatologia e Nutrição Pediátrica (NASPGHAN): quando o resultado da bilirrubina total (BT) sérica for maior do que $5,0 \mathrm{mg} / \mathrm{dL}$ e a bilirrubina direta (BD) apresentar-se acima de $20 \%$ do valor da BT, ou se a bilirrubina total for menor do que $5,0 \mathrm{mg} / \mathrm{dL}$, porém a bilirrubina direta estiver acima de $1,0 \mathrm{mg} / \mathrm{dL}^{(22)}$.

\section{Antropometria}

Os dados antropométricos foram obtidos pelo mesmo observador (Schneider ACR). Foram verificados peso, altura, circunferência braquial (CB), prega cutânea tricipital (PCT), circunferência muscular do braço $(\mathrm{CMB})$ e índice de massa corpórea (IMC). Todos os pacientes abaixo de 1 ano foram pesados sem roupa em balança digital com capacidade até $16 \mathrm{~kg}$. Os maiores foram pesados com roupas leves em balança manual Filizola com capacidade até $150 \mathrm{~kg}$. As medidas de CB e PCT foram tomadas em triplicata, a meia distância entre o olécrano e o acrômio, sendo utilizada a média de cada verificação. Para medir a CB foi utilizada fita métrica flexível, inelástica e para a PCT o adipômetro científico de fabricação nacional (Cescorf). A CMB foi calculada segundo a fórmula de FRISANCHO ${ }^{(12)}$. $\mathrm{CMB}(\mathrm{mm})=\mathrm{CB}(\mathrm{mm})-3,14 \mathrm{X}$ PCT.

O IMC foi calculado para crianças acima de 24 meses através do resultado da divisão do peso em kilogramas, pela estatura ao quadrado em metros.

Os índices antropométricos peso para idade $(\mathrm{P} / \mathrm{I})$, estatura para idade (E/I) e índice de massa corporal (IMC) foram calculados pelo escore $Z$. Por este escore determina-se que pacientes em risco nutricional encontram-se no escore $Z \leq-1,28$ (correspondente ao percentil 10) e os desnutridos apresentam escore $Z \leq-2,0$ (percentil 3) ${ }^{(19,34)}$. O escore $Z=0$, correspondente ao percentil 50 , indicando a média da população normal na mesma faixa etária de acordo com a tabela de referência do National Center for Health Statistics (NCHS).

A espessura de prega cutânea tricipital (PCT) e a circunferência muscular do braço (CMB) foram comparadas com os valores normais das tabelas de FRISANCHO ${ }^{(13)}$ em relação ao sexo e idade e também foi utilizado como ponto de corte para risco nutricional o percentil $\leq 10$ e desnutrição o percentil $\leq 3$.

\section{Considerações éticas}

Este estudo foi aprovado pela Comissão de Pesquisa em Ética e Saúde do HCPA e todos os responsáveis pelos pacientes assinaram o termo de consentimento informado.

\section{Estatística}

A análise dos dados foi feita com auxílio do programa NutStat (EPIINFO, versão 3.2, CDC/WHO 2000). Em relação à idade e aos índices $\mathrm{P} / \mathrm{I}$, E/I e IMC os dados apresentaram distribuição simétrica (testes Kolmogorov-Smirnov e Shapiro-Wilk) e foram apresentados em média e desvio padrão. Os índices PCT e CMB apresentaram distribuição assimétrica e foram apresentados em mediana.

\section{RESULTADOS}

As características dos pacientes estão apresentadas na Tabela 1. Os fatores etiológicos de cirrose determinados foram os seguintes: atresia biliar em 15 pacientes, hepatite auto-imune em 11 , fibrose cística em 3 , deficiência de $\alpha 1$-antitripsina em 1 e histiocitose de Langerhans em 1 paciente. Dos 11 pacientes com doença auto-imune, 3 foram avaliados antes de iniciarem o tratamento com corticóides.

Dos pacientes que participaram do estudo, 17 apresentaram colestase. Destes, 6 pacientes estavam abaixo do ponto de corte para risco nutricional para os índices estudados.

A média de $\mathrm{P} / \mathrm{I}$ de todos os pacientes investigados foi de - 0,38 $\pm 1,40$ DP (amplitude de -5,2 a 2,15), a média da E/I foi de $-0,83 \pm 1,16$ DP $(-3,92$ a 1,76$)$ e do IMC foi de $0,17 \pm 1,3 \mathrm{DP}$ $(-3,1$ a 1,97). Observa-se nas Figuras 1 e 2, que a maioria dos 
TABELA 1. Características de 42 crianças e adolescentes com cirrose

\begin{tabular}{lc}
\hline Idade (meses) & \\
$\quad$ Mediana (amplitude) & $99 \mathrm{~m}(3 \mathrm{~m}-216 \mathrm{~m})$ \\
\hline Sexo & \\
Masculino & $13(31 \%)$ \\
Feminino & $29(69 \%)$ \\
Fatores etiológicos & \\
Atresia biliar & $15(36 \%)$ \\
Hepatite auto-imune & $11(26 \%)$ \\
Criptogênica & $11(26 \%)$ \\
Outros & $5(12 \%)$ \\
Classificação de Child-Pugh & \\
A & $22(52 \%)$ \\
B & $15(36 \%)$ \\
C & $5(12 \%)$ \\
Colestase & $17(40,5 \%)$ \\
\hline
\end{tabular}

pacientes encontrou-se abaixo da média populacional para os índices de $\mathrm{P} / \mathrm{I}(23 / 42,54,8 \%)$ e E/I (32/42, 76,2\%). Para o índice $\mathrm{P} / \mathrm{I}$ encontraram-se $3 / 42(7,1 \%)$ pacientes em risco nutricional e 6/42 (14,3\%) em estado de desnutrição. Em relação a E/I, 8/42 (19\%) pacientes apresentaram risco nutricional e 7/42 (16,7\%) já apresentaram crescimento comprometido por desnutrição. Ao determinar-se o escore $\mathrm{Z}$ do IMC, foi detectado comprometimento nutricional em $5 / 38(13,2 \%)$ pacientes, estando por este índice $2 / 38(5,3 \%)$ pacientes em risco e $3 / 38(7,9 \%)$ desnutridos.

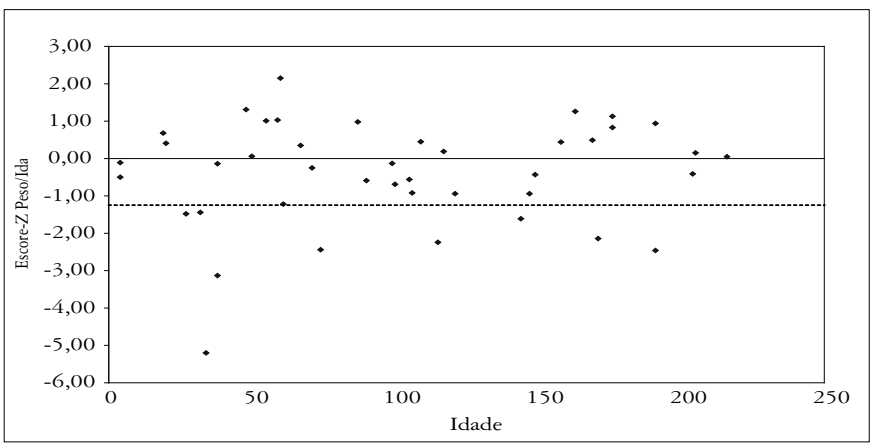

FIGURA 1. Peso/idade. A linha reta corresponde ao escore $Z=0$ e a linha pontilhada, ao escore $Z=-1,28$. Observa-se que $23 / 42(54,8 \%)$ encontra-se abaixo do escore $Z=0$ e $9 / 42(21,4 \%)$ pacientes encontram-se abaixo do escore $Z-1,28$

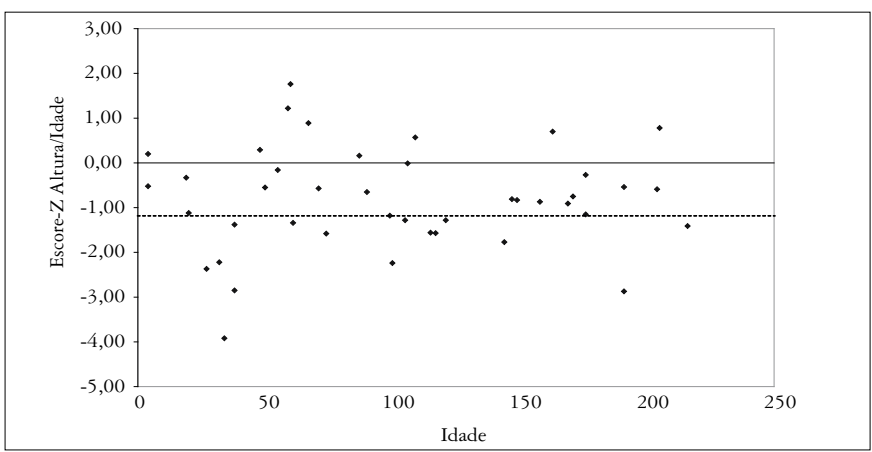

FIGURA 2. Estatura/idade. A linha reta corresponde ao escore $Z=0$ e a linha pontilhada ao escore $Z=-1,28$. Observa-se que $32 / 42(76 \%)$ dos pacientes encontramse abaixo do escore $Z=0$ e $15 / 42(35,7 \%)$ abaixo do escore $Z$ - 1,28
As avaliações de PCT e CMB foram obtidas de 37 pacientes. Os pacientes encontrados abaixo do percentil 50, foram respectivamente $24 / 37$ (65\%) e 20/37 (54\%). Ao se utilizar o ponto de corte para risco nutricional, os pacientes em risco eram $12 / 37(32,4 \%)$ para a PCT e $9 / 37(24,3 \%)$ para a CMB. Foram considerados desnutridos $4 / 37$ pacientes $(10,8 \%)$, tanto pela PCT como pela CMB. As medianas das medidas de PCT e CMB estiveram no percentil 25 com amplitude do percentil 3 ao 90 .

\section{DISCUSSÃO}

Apesar da desnutrição grave ser facilmente reconhecida, a distinção entre nutrição adequada e desnutrição leve a moderada não é tão clara. Alguns estudos na literatura consideram inadequado qualquer índice nutricional encontrado abaixo do valor de referência da população normal ${ }^{(11,31)}$. A implementação da terapia nutricional mais adequada depende da avaliação do estado nutricional do paciente. A Organização Mundial de Saúde (OMS), considera desnutridas as pessoas com índices antropométricos inferiores a -2 desvios-padrão abaixo da média de referência. Indivíduos com índices entre os desvios-padrão $-1,28$ a $-2,0$ do escore $Z$ estão em risco nutricional, portanto momento adequado a intervenção visando a sua recuperação ${ }^{(34)}$. O comprometimento do estado nutricional é um fator de risco para aumento da morbidade e mortalidade nas hepatopatias, portanto o constante e cuidadoso monitoramento do paciente é fundamental ${ }^{(31)}$.

Índices que envolvam o peso corporal não são os mais adequados para constatação de comprometimento nutricional nos hepatopatas crônicos, porém o acompanhamento regular do P/I é importante para observação de dificuldade de ganho de peso ou perdas agudas. Foram detectados nove pacientes abaixo do ponto de corte considerado como de risco, destes três se encontravam em risco nutricional e seis desnutridos. Pode-se conjecturar que se não houvesse a interferência da visceromegalia e, em alguns casos, da retenção hídrica ocasionada por ascite, edema e/ou uso de corticóides, talvez o número de pacientes em risco nutricional fosse mais elevado.

Na Figura 3 são apresentados resultados obtidos em estudos com crianças e adolescentes com doença hepática crônica. Assim como no presente estudo, os desvios-padrão para os índices P/I e E/I são muito altos, o que denota grande variação dos valores individuais. Há crianças hepatopatas crônicas, com o estado nutricional adequado e outras muito comprometidas. Pode-se observar a heterogeneidade de achados em relação aos mesmos índices utilizados. Por exemplo, no estudo de CHIN et al. ${ }^{(9)}$, onde foram avaliadas crianças com doença hepática em estado terminal, as médias de $\mathrm{P} / \mathrm{I}$ e $\mathrm{E} / \mathrm{I}$ se encontravam no escore $\mathrm{Z}-2,0$, indicando número considerável de crianças bastante depletadas nutricionalmente. No presente estudo e em outros ${ }^{(14,31)}$ com hepatopatas compensados, considerando as médias dos mesmos índices, não se encontraram amostras com alterações tão significativas. 
TABELA 2. Estudos com avaliação nutricional em crianças com hepatopatias crônicas

\begin{tabular}{|c|c|c|c|c|}
\hline Autor e ano & $\mathrm{n}$ & Pacientes & Idades & $\begin{array}{c}\text { Resultados } \\
\text { (escore } \mathrm{Z} \text { ) } \\
\overline{\mathbf{X}}_{ \pm \mathrm{DP}^{*}}\end{array}$ \\
\hline $\begin{array}{l}\text { Hambidge et al., } \\
1987^{(18)}\end{array}$ & 45 & $\begin{array}{l}\text { Hepatopatas } \\
\text { crônicos (AVB) }\end{array}$ & Crianças & $\begin{array}{l}\mathrm{P} / \mathrm{I}-1,58 \pm 1,67 \\
\mathrm{E} / \mathrm{I}-1,44 \pm 1,74\end{array}$ \\
\hline Sokoll e Stall, $1990^{(31)}$ & 56 & $\begin{array}{l}\text { Hepatopatas } \\
\text { crônicos }\end{array}$ & 1 mês -10 anos & $\begin{array}{l}\mathrm{P} / \mathrm{I}-0,61 \pm 1,08 \\
\mathrm{E} / \mathrm{I}-0,93 \pm 1,36\end{array}$ \\
\hline Chin et al., $1992^{(9)}$ & 27 & $\begin{array}{l}\text { Hepatopatas } \\
\text { crônicos }\end{array}$ & 5 meses $-6,7$ anos & $\begin{array}{l}\mathrm{P} / \mathrm{I}-2,0 \pm 1,36 \\
\mathrm{E} / \mathrm{I}-2,53 \pm 1,54\end{array}$ \\
\hline Gazal, $1995^{(14)}$ & 26 & Cirróticos & 9 meses -17 anos & $\begin{array}{l}\mathrm{P} / \mathrm{I}-0,76 \pm 1,34 \\
\mathrm{E} / \mathrm{I}-1,13 \pm 1,79\end{array}$ \\
\hline Cardoso et al., $1997^{(6)}$ & 11 & $\begin{array}{l}\text { Hepatopatas } \\
\text { crônicos } \\
\text { (colestáticos) }\end{array}$ & 5 meses $-8,7$ anos & $\begin{array}{l}\mathrm{P} / \mathrm{I}-1,19 \pm 1,14 \\
\mathrm{E} / \mathrm{I}-1,51 \pm 1,11\end{array}$ \\
\hline Presente estudo & 42 & Cirróticos & 4 meses -18 anos & $\begin{array}{l}\mathrm{P} / \mathrm{I}-0,38 \pm 1,40 \\
\mathrm{E} / \mathrm{I}-0,83 \pm 1,16\end{array}$ \\
\hline
\end{tabular}

$\mathrm{n}=$ número de pacientes

$\bar{X} \pm D P=$ média \pm desvio-padrão

CHIN et al. ${ }^{(9)}$ mostraram ainda que o índice E/I foi o que apresentou o maior número de crianças abaixo do ponto de corte para desnutrição de $-2,0 \mathrm{Z}$. Em outros estudos (Tabela 2), com exceção do de HAMBIDGE et al..$^{(18)}$, pode-se perceber que este índice é o mais afetado em crianças com hepatopatias. Este déficit mais acentuado no crescimento, indica a presença de comprometimento nutricional de longa duração o qual afetou o ganho estatural, contudo a retomada do crescimento pode ser alcançada com orientação nutricional adequada ou após o transplante hepático ${ }^{(26,33)}$.

A medida de CMB estima a reserva protéica e a PCT a reserva de gordura. A PCT é uma medida que apresenta boa correlação com a reserva adiposa corporal e em conjunto com a CMB, são métodos sensíveis a mudanças no estado nutricional ${ }^{(23,27,32)}$. Estes parâmetros são menos influenciáveis pelas manifestações clínicas das hepatopatias ${ }^{(31)}$. Nos pacientes avaliados, o índice que melhor refletiu o risco nutricional foi a espessura de PCT. Alguns pacientes, apresentaram valores de $\mathrm{P} / \mathrm{I}$ dentro da normalidade $\mathrm{e}$ PCT diminuído, o que demonstra que o acompanhamento desta medida pode indicar precocemente o risco nutricional. Outros estudos $^{(27,31)}$, também observaram que medidas que estimam reservas calóricas e protéicas se encontram mais deprimidas do que outros parâmetros nutricionais nos hepatopatas. Vários fatores contribuem para a desnutrição calórico-protéica e deficiências de vitaminas e minerais na cirrose ${ }^{(29)}$ : a ingestão pode ser insuficiente devido à anorexia, náuseas, vômitos, saciedade precoce, mudanças de paladar provocadas por medicamentos ou por distúrbios bioquímicos e também por causa de dificuldades comportamentais em relação à alimentação; os comprometimentos da digestão e absorção podem ser ocasionados por redução do fluxo biliar, causando a má absorção de gorduras, vitaminas lipossolúveis e deficiência de ácidos graxos essenciais e pela enteropatia devido à hipertensão portal. Os hepatopatas também podem ter necessidades nutricionais aumentadas por causa de hipermetabolismo ${ }^{(16)}$, infecções freqüentes e síntese insuficiente de proteínas. GREER et al. ${ }^{(17)}$ observaram a presença de hipermetabolismo em crianças com doença hepática avançada. Este achado implica na importância da orientação nutricional a fim de minimizar os efeitos do estado catabólico. $\mathrm{O}$ gasto metabólico aumentado contribui para a depleção de reservas adiposas e protéicas, por este motivo, para estes pacientes, a recomendação de calorias é definida em cerca de $30 \%$ a $50 \%$ acima da $\mathrm{RDA}^{(3,4,23)}$. Frente aos problemas de ingestão, digestão e absorção anteriormente mencionados e devido à necessidade de maior aporte de calorias e nutrientes, é difícil alcançar o valor energético demandado pelo paciente e há necessidade da inclusão de fórmulas e suplementos para facilitar o manejo dietoterápico. A deficiência protéico-calórica de nutrientes específicos e de micronutrientes como zinco, ferro e selênio, estão associados com o déficit de crescimento e à diminuição da síntese de proteínas e devem ser prevenidos ou tratados.

Outro índice antropométrico avaliado nos pacientes do atual estudo foi o IMC. Constatou-se que somente cinco pacientes apresentaram comprometimento nutricional por este índice, que não demonstrou ser um método adequado para avaliar cirróticos por superestimar o seu estado nutricional.

$\mathrm{Na}$ presente série, por apresentar um número pequeno de pacientes com risco nutricional e desnutridos, não foi possível realizar associações conclusivas entre os índices antropométricos e a gravidade da cirrose e com a presença de colestase, entretanto em outros estudos de avaliação nutricional com pacientes pediátricos cirróticos ${ }^{(6,14)}$ também não foram observadas tais associações. Em relação à presença de colestase, neste estudo observou-se que 11/17 pacientes com colestase apresentaram estado nutricional adequado. No estudo de BASTOS e SILVEIRA ${ }^{(2)}$ não foi observada relação entre a desnutrição determinada pela antropometria com a colestase, porém esta relação foi estabelecida no estudo de MATTAR et al. ${ }^{(21)}$.

A inclusão de pacientes com uso de corticóides merece algumas considerações, já que estes pacientes freqüentemente apresentam retenção hídrica. Estimativas da reserva de gordura corporal pela PCT e da proteína pela CMB são melhores indicadores do estado nutricional, mesmo na presença de retenção de fluidos, porque o edema ocorre em menor extensão nos membros superiores ${ }^{(3)}$.

Em conclusão, observaram-se importantes alterações, considerando o risco nutricional das crianças e adolescentes avaliados, especialmente relacionados aos índices antropométricos PCT e CMB. O índice E/I foi o que apresentou maior número de pacientes comprometidos nutricionalmente para o escore Z -2,0. Devido a importância do estado nutricional em pacientes com doença hepática crônica, recomenda-se o acompanhamento destes pacientes, com a realização de avaliações seriadas com ênfase na utilização de medidas que avaliem a composição corporal e determinem precocemente o risco nutricional, principalmente a PCT. 
Schneider ACR, Pinto RB, Silveira TR. Nutritional risk and malnutrition determination by anthropometry in cirrhotic children and adolescents. Arq Gastroenterol. 2007;44(4):345-9.

ABSTRACT - Background - The malnutrition is a frequent finding in adults with cirrhosis, but the prevalence of nutritional risk and malnutrition is little known in pediatric patients. Aim - To evaluate through anthropometry the presence of nutritional risk and malnutrition in cirrhotic pediatric patients regularly attended at the Pediatric Gastroenterology Service of "Hospital de Clínicas" of Porto Alegre, RS, Brazil. Methods - Cross-sectional study with 42 cirrhotic children and adolescents aged between 3 months and 18 years. The nutritional evaluation was made by the determination of the weight/age, height/age, body mass index and triceps skinfold thickness and arm muscle circumference measurements. Patients considered in nutritional risk were $\leq-1,28 \mathrm{Z}$ score which corresponds to $\leq 10$ th percentile, and those under $-2,0 \mathrm{Z}$ and $\leq 3$ th percentile were in malnutrition status. According to Child-Pugh criteria, 22 patients were classified as A (mild severity), 15 (moderate) B and $5 \mathrm{C}$ (intense). Results - The mean weight/age, height/age and body mass index $\mathrm{Z}$ scores were, respectively, - $0,38 \pm 1,4$ $\mathrm{SD},-0,83 \pm 1,16 \mathrm{SD}$ and $0,17 \pm 1,3 \mathrm{SD}$. Patients in nutritional risk were 3/42 (weight/age), 8/42 (height/age), $12 / 37$ (triceps skinfold thickness), $9 / 37$ (arm muscle circumference), $2 / 38$ (body mass index); in malnutrition status were 6/42 (weight/age), 7/42 (height/age), 4/37 (triceps skinfold thickness) and 4/37 (arm muscle circumference) and 3/38 (body mass index). Conclusion - The prevalence of nutritional risk was $32.4 \%$ and chronic malnutrition was $16.7 \%$. The index which better reflected the nutritional risk in these patients was triceps skinfold thickness. Chronic malnutrition status occurrence was greater in the height/age index.

HEADINGS - Child nutrition disorders. Nutritional status. Anthropometry. Liver cirrhosis. Adolescent. Child.

\section{REFERÊNCIAS}

1. Alvares-da-Silva MR, Silveira TR. Comparison between handgrip strength, subjective global assessment, and prognostic nutritional index in assessing malnutrition and predicting clinical outcome in cirrhotic outpatients. Nutrition. 2005;21:113-7.

2. Bastos MD, Silveira TR. Níveis plasmáticos de vitamina $\mathrm{D}$ em crianças e adolescentes com colestase. J Pediatr. 2003;79:245-52.

3. Bavdekar A, Bhave S, Pandit A. Nutrition management in chronic liver disease. Indian J Pediatr. 2002;69:427-31.

4. Brook G, Hunt J, Johnson A, Reed J. Supporting the child and family. In: Kelly DA, editor. Diseases of the liver and biliary system in children. Oxford: Blackwell Publishing; 2004. p.1-16.

5. Campillo B, Richardet JP, Scherman E, Bories PN. Evaluation of nutritional practice in hospitalized cirrhotic patients: results of a prospective study. Nutrition. 2003;19:515-21.

6. Cardoso AL, Porta G, Vieira MA, Carraza FR. Caracterização nutricional de crianças com colestase crônica. J Pediatr (Rio J). 1997;73:43-50.

7. Caregaro 1, Alberino F, Amodio P, Merkel C, Bolognese M, Angeli P, Gatta A. Malnutrition in alcoholic and virus related cirrhosis. Am J Clin Nutr. 1996;63:602-9.

8. Carvalho L, Parise ER. Avaliação em ambulatório do estado nutricional em pacientes com cirrose hepática. Arq Gastroenterol. 2006;43:269-74.

9. Chin SE, Shepherd RW, Thomas BJ, Cleghorn, GJ, Patrick MK, Wilcox JA. The nature of malnutrition in children with end stage liver disease awaiting for orthotopic liver transplantation. Am J Clin Nutr. 1992;56:164-8.

10. Figueiredo FA, de Mello Perez R, Kondo M. Effect of liver cirrhosis on body composition: evidence of significant depletion even in mild disease. J Gastroenterol Hepatol. 2005;20:209-16.

11. Foster BJ, Leonard MB. Measuring nutritional status in children with chronic kidney disease. Am J Clin Nutr. 2004;80:801-14.

12. Frisancho RA. New norms of upper limb fat and muscle areas for assessment of nutritional status. Am J Clin Nutr. 1974;34:2540-4.

13. Frisancho RA. Triceps skin fold and upper arm muscle size norms for assessment of nutritional status. Am J Clin Nutr. 1981;27:1052-8.

14. Gazal CH. O inquérito nutricional, a antropometria e a avaliação da eliminação intestinal de proteínas e gorduras pela dosagem da alfa 1 antitripsina fecal e do esteatócrito na avaliação nutricional de crianças com cirrose. [Dissertação] Curso de Pós-Graduação em Ciências Médicas, Pediatria da Universidade Federal do Rio Grande do Sul; 1995.

15. Gottschall CB, Alvares-da-Silva MR, Camargo AC, Burtett RM, Silveira TR. Avaliação nutricional de pacientes com cirrose pelo vírus da hepatite $\mathrm{C}$ : a aplicação da calorimetria indireta. Arq Gastroenterol. 2004;41:220-4.

16. Greco AV, Mingrone G, Benedetti G, Capristo E, Tataranni PA, Gasbarrini G Daily energy and substrate metabolism in patients with cirrhosis. Hepatology. 1998;27:346-50.

17. Greer R, Lehnert M, Lewindon P, Cleghorn GJ, Shepherd RW. Body composition and components of energy expenditure in children with end-stage liver disease. J Pediatr Gastroenterol Nutr. 2003;36:358-63.
18. Hambidge KM, Krebs NF, Lilly G, Zerbe GO. Plasma and urine zinc in infants and children with extrahepatic billiary atresia. J Pediatr Gastroenterol Nutr. 1987;6:872-7.

19. Maio R, Dichi JB, Burini RC. Sensibilidade de indicadores da desnutrição protéicoenergética em cirróticos com vários graus de disfunção hepatocelular. Arq Gastroenterol. 2004;41:93-9.

20. Maio R, Dichi JB, Burini RC. Consequências nutricionais das alterações metabólicas dos macronutrientes na doença hepática crônica. Arq Gastroenterol. 2000;37:52-7.

21. Mattar RH, de Azevedo RA, Speridião PG, Neto UF, Morais MB. Estado nutricional e absorção intestinal de ferro em crianças com doença hepática crônica com e sem colestase. J Pediatr (Rio J). 2005;81:317-24.

22. North American Society for Pediatric Gastroenterology, Hepatology and Nutrition. Guideline for the evaluation of cholestatic jaundice in infants: recommendations of the North American Society for Pediatric Gastroenterology, Hepatology and Nutrition. J Pediatr Gastroenterol Nutr. 2004;39:115-28.

23. Novy MA, Schwarz KB. Nutritional considerations and management of the child with liver disease. Nutrition. 1997;13:177-84.

24. Panagaria N, Varma K, Nijhaman S, Mathur A, Rai RR. Comparison of nutritional status between patients with alcoholic and non-alcoholic liver cirrhosis. Trop Gastroenterol. 2006;27:75-9.

25. Pugh NH, Murray-Lyon IM, Dawson JL, Pietroni MC, Williams R. Transection of the oesophagus varices. Br J Surg. 1973;60:646-9.

26. Ramaccioni V, Soriano HE, Arumugan R, Klish WJ. Nutritional aspects of chronic liver disease and liver transplantation in children. J Pediatr Gastroenterol Nutr. 2000;30:361-7.

27. Roggero P, Cataliotti E, Ulla L, Stuflesser S, Nebbia G, Bracaloni D, Lucianetti A, Gridelli B. Factors influencing malnutrition in children waiting for liver transplants. Am J Clin Nutr. 1997;65:1852-7.

28. Roongpisuthipong C, Sobhonlidsuk A, Nantiruj K, Songchitsomboon S. Nutritional assessment in various stages of liver cirrhosis. Nutrition. 2001;17:761-5.

29. Shepherd RW. Management of chronic liver disease. In: Kelly DA, editor. Diseases of the liver and biliary system in children. Oxford: Blackwell; 2004. p.259-81.

30. Sigulem DM, Devicenzi UM, Lessa AC. Diagnóstico do estado nutricional da criança e do adolescente. J Pediatr (Rio J). 2000;73:s275-s84.

31. Sokol RJ, Stall C. Anthropometric evaluation of children with chronic liver disease. Am J Clin Nutr. 1990;52:203-8.

32. Taylor RM, Dhawan A. Assessing nutritional status in children with chronic liver disease. J Gastroenterol Hepatol. 2005;20:1817-24.

33. Viner RM, Forton JTM, Cole TJ, Clark IH, Noble-Jamieson G, Barnes ND. Growth of long term survivors of liver transplantation. Arch Dis Child. 1999;80:235-40.

34. WHO - World Health Organization Working Group. Use and interpretation of anthropometric indicators of nutritional status. Bull WHO. 1986;64:929-41. 